\title{
Immunological Barriers to Stem-Cell Based Cardiac Repair
}

\author{
Zaruhi Karabekian • Nikki Gillum Posnack • \\ Narine Sarvazyan
}

Published online: 18 November 2010

(C) The Author(s) 2011. This article is published with open access at Springerlink.com

\begin{abstract}
Repair of damaged myocardium with pluripotent stem cell derived cardiomyocytes is becoming increasingly more feasible. Developments in stem cell research emphasize the need to address the foreseeable problem of immune rejection following transplantation. Pluripotent stem cell (PSC) derived cardiomyocytes have unique immune characteristics, some of which are not advantageous for transplantation. Here we review the possible mechanisms of PSC-derived cardiomyocytes rejection, summarize the current knowledge pertaining to immunogenicity of such cells and describe the existing controversies. Myocardial graft rejection can be reduced by modifying PSCs prior to their differentiation into cardiomyocytes. Overall, this approach facilitates the development of universal donor stem cells suitable for the regeneration of many different tissue types.
\end{abstract}

Keywords Embryonic stem cells · Induced pluripotent stem cells $\cdot$ Cardiomyocytes $\cdot$ Differentiation $\cdot$ Immunogenicity

\section{Introduction: Stem Cells in Cardiac Repair}

Myocardial infarction remains a major cause of morbidity and mortality worldwide. This is an unmet clinical demand to repair irreversibly damaged myocardial tissue. The mitotic capacity of heart muscle cells is limited and insufficient to support cardiac regeneration [1-3]. Therefore, injured cardiac myocytes are replaced by fibroblasts

Z. Karabekian $\cdot$ N. G. Posnack $\cdot$ N. Sarvazyan $(\bowtie)$

Pharmacology and Physiology Department,

The George Washington University,

2300 Eye Street,

Washington, DC 20037, USA

e-mail: phynas@gmail.com and connective tissue, resulting in the formation of an arrhythmogenic scar. The remaining cells enlarge to compensate for the additional workload, leading to hypertrophy, and eventually to heart failure. Today, stem cells are actively studied to engineer functional excitable systems that can be grafted to injured myocardium following infarction [4-7]. In addition, stem cells also hold the promise of repairing injured cardiac pacemaker or conductive system pathways $[8,9]$.

\section{Major Hurdles to Successful Stem Cell Based Cardiac Repair}

Many studies have attempted to use stem cell-derived cardiomyocytes to aid the functional recovery of infarcted myocardium. While there is plenty of evidence of improved cardiac function following stem cell transplantation, the exact mechanisms behind these improvements are actively debated [10]. Clinical implementation of stem cell therapy will require a better understanding of these mechanisms. In addition, a number of technical and physiological problems have to be solved. Currently recognized hurdles of stem cell therapy for cardiac repair can be grouped into the following categories: teratoma formation, graft arrhythmogenicity, vascularization of the graft, acute and long-term donor cell viability, integration into the host anatomical structure and immune rejection of the graft. In this review we will focus on immune rejection of stem cell-based cardiac grafts transplanted into the heart of either allogeneic or xenogeneic hosts (allogeneic - derived from different individuals of the same species, xenogeneic - from an organism of a different species). While the immunogenicity of stem cells is often mentioned as a potential caveat, few studies have tried to experimentally address it. As of today, the majority 
of studies on stem cell-based myocardial grafts derive their conclusions from experiments conducted in severely immunosuppressed or immunodeficient hosts [11, 12]. The goal of this review is to explain the immunological barriers of stem cell based cardiac repair and the ways these barriers may be overcome. It is especially important since an ultimate destination of transplanted cells is the injured myocardium, and the inherent inflammatory environment of injured tissue is expected to further aggravate the immune reaction of the host.

\section{Basic Immunology of Graft Rejection}

Transplantation of a graft between genetically unrelated organisms typically results in a robust immune response and consequently, graft rejection. T-lymphocytes recognize foreign antigens in the form of peptides, which are presented in association with self-MHC molecules. There are two primary classes of MHC molecules. MHC class I antigens are found on the surface of every nucleated cell; they display protein fragments synthesized within the cell to Cytotoxic T Lymphocytes (CTL), or $\mathrm{CD} 8^{+} \mathrm{T}$ cells. Cell surface expression of MHC I antigens is the predominant reason for immune detection and rejection of allogeneic grafts. In comparison, MHC class II antigens are expressed only on a few specialized cell types, including macrophages, dendritic cells and B lymphocytes. These cells are called professional antigen-presenting cells. The function of MHC II molecules is to display peptides of exogenous proteins to T-helper cells, or $\mathrm{CD} 4^{+} \mathrm{T}$ cells.

Direct allorecognition arises when both types of host T-lymphocytes $\left(\mathrm{CD}^{+}\right.$and $\left.\mathrm{CD}^{+}\right)$are stimulated by donor antigen presenting cells. This stimulation occurs through a direct interaction between host T-lymphocyte receptors and MHC I \& II antigens expressed on the surface of donor cells (Fig. 1).

Indirect allorecognition occurs when donor MHC peptides are processed and then presented by host MHC II molecules. Host antigen presenting cells internalize and degrade donor MHC peptides, display those peptides onto their MHC II molecules and present them to host $\mathrm{CD} 4^{+} \mathrm{T}$ cells (Fig. 1). This process triggers an immune response against grafts expressing both donor MHC I and II. All-inall, the presence of MHC molecules is the defining factor that largely determines the degree of graft rejection.

There are three phases of acquired immune rejection. Hyperacute rejection occurs within minutes of transplantation. In this case, rejection is induced by pre-existing host antibodies that bind to the graft's antigens. This binding activates the complement system and a sequence of events ensues, including: an influx of peripheral blood mononuclear cells, formation of platelet thrombi, small vessel thrombosis,

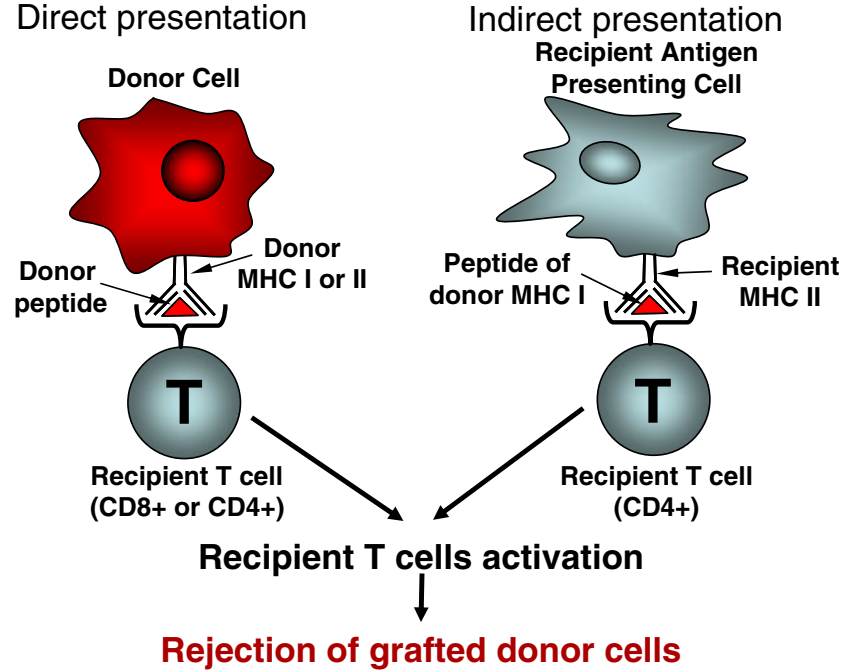

Fig. 1 Schematic representation of direct and indirect recognition of donor antigens to the recipient $\mathrm{T}$ cells

and finally, damage and/or destruction of the graft. Acute rejection occurs within 1-2 weeks after transplantation and is characterized by capillary rupture and severe graft infiltration by monocytes/macrophages, lymphocytes and dendritic cells. This hypersensitive reaction is mediated by Cytotoxic T lymphocytes. Chronic rejection occurs months or years after transplantation. This reaction is associated with deposits of immunoglobulin and $\mathrm{C} 3$ complement molecules on the basement membrane of graft cells.

When using stem cell derived grafts, all three phases of rejection should be considered.

\section{Potential Cell Sources}

a) Allogeneic vs syngeneic cells. Syngeneic grafts made from cells genetically identical to the host remain an attractive therapeutical goal due to their minimal immunological implications. This is an active area of research that includes the use of somatic cell nuclear transfer protocols [13] and the derivation of pluripotent adult stem cells and multipotent progenitor cells from target tissues $[14,15]$. It also includes the attempt to derive a patient's own embryonic-like stem cells via genetic reprogramming of differentiated somatic cells $[16,17]$. These cells are called induced pluripotent stem cells or iPS cells. iPS cells of both mouse and human origin have been successfully differentiated into cardiomyocytes [16-18]. Albeit a very promising future source to generate patient-specific grafts, iPS-based therapies have their own set of problems. These include the low reliability of the dedifferentiation protocol, the large quantity of cells necessary for clinical use and high costs associated with customized therapy. In the case of 
chronic degenerative diseases, treating patients with cells derived from their own somatic cells will do little more than perpetuate the existing pathology. Long time delays associated with iPS cells derivation and proliferation can be problematic for clinical applications in which a large amount of cells is needed within a short period of time following the injury. Therefore, allogeneic stem cellderived cells remain an important transplantation source.

b) Adult vs embryonic stem cells. Adult stem cells from different tissues can reportedly produce cells with a cardiomyocyte-like phenotype [15]. In addition, several studies indicate the presence of so-called cardiac resident stem cells that can give rise to functional cardiomyocytes [2, 19-21]. However, cardiac resident stem cells are rare. They are difficult to identify, extract and proliferate to the amounts necessary for cardiac tissue regeneration. Thus far, the most reliable in vitro sources used to generate cardiomyocytes are embryonic stem cells (ESC) or cells returned to an ESC-like state, the iPS cells mentioned above. ESC are derived from the inner cell mass of the blastocyst of a developing embryo [22-25]. They retain the ability to differentiate into a multitude of specialized cell types, given the appropriate conditions [26, 27]. In the case of iPS cells, the expression of key transcription factors, such as Oct-4, Sox2, NANOG, c-Myc, KLF4 and LIN28, has been shown to revert somatic cells to a pluripotent, ESC-like state $[16,28]$. These ground-breaking studies used retroviral-driven expression, which is considered to be one of the most efficient methods for reverting somatic cells, yet it can result in neoplastic transformation. Incorporating transgenes into the genome could also increase the number of immunogenic antigens of future graft cells. To circumvent these problems, alternative methods are being developed to reprogram somatic cells. These include using the piggyBac transposon system [29] and non-viral transfection via single multiprotein expression vector [30] to deliver reprogrammable elements. The latest technique of re-differentiation of adult somatic cells relies on synthetic modified mRNA molecules to deliver reprogramming signals [31]. These approaches minimize the risk of neoplastic conversion and the immunological implications of trangene expression.

All-in-all, it is apparent that other cell types suitable for regenerative purposes are likely to be discovered or created. Yet, as of today, cells that exhibit significant potential for tissue regeneration are cells with the pluripotent stem cell (PSC) phenotype. These can originate from an embryoembryonic stem cells (ESC), or an adult organism-adult stem cells, adult progenitor cells, and induced pluripotent stem cells (iPS). For the purposes of this review we will be using the collective term Pluripotent Stem Cells (PSC) whenever the origin of pluripotent cells is not important.

\section{Immunological Implications of Methods Used to Produce Stem Cell-Derived Cardiac Myocytes}

Currently there are four main methods by which cardiomyocytes are derived from PSCs:

a) the hanging drop method, b) exposure to biologically active molecules or cocktail-based protocol, c) genetic selection and d) non-genetic enrichment. Since the immunogenicity of stem cell-derived grafts is influenced by the method used to direct cell differentiation, these four methods will be discussed below in more detail.

a) The hanging-drop method is used to create a pseudoembryo, termed an embryoid body (EB) [32, 33]. These round masses of cells spontaneously form contracting cardiomyocytes as a part of their heterogeneous cell population $[34,35]$. The cardiomyocytes found within EBs follow a differentiation pattern that mimics that of early myocardium development, with GATA-4 and Nkx 2.5 transcription factors expressed prior to cardiac specific cytoplasmic proteins, such as $\beta$-myosin heavy chain, $\alpha$-actinin and $\alpha$-actin $[36,37]$. Many labs, including our own [38], use EB-differentiation to produce clusters of beating cells followed by cell enrichment protocols, which are based on density differences or cardiac lineage fluorescent markers. The main disadvantages of utilizing the hanging-drop method is low yield in cells of interest (less than 10\%) and the need for subsequent purification. The main immunological concern is that some of the cells within a growing EB will unavoidably differentiate into professional antigen presenting cells. These cells can significantly increase the rejection of EB derived grafts.

b) Exposure to biologically active molecules allows one to increase the quantity of derived cardiomyocytes with or without the EB step [39]. A variety of compounds have been shown to effectively induce cardiomyocyte differentiation, including: ascorbic [40] and retinoic acids [41], BMP4 and activin A [39], and a cocktail of recombinant molecules [42]. The success of these protocols is dependent upon strict timing at a defined concentration to increase the quantity of derived cardiomyocytes. These techniques reportedly lead to significant enrichment, typically within the range of $30-50 \%$ cardiomyocytes in the total cell population. These numbers are often hard to reproduce because enrichment depends on the initial stage of undifferentiated ESC. The immunogenicity of myocytes derived by cocktail-based protocols is expected to be similar to 
those obtained though EB formation, although the number of professional antigen presenting cells is likely to be smaller.

The EB-based and the cocktail-type method result in a heterogeneous cell population, which may include a subpopulation of professional antigen presenting cells [43]. The additional purification step that is required also leads to reduced viability and low cardiomyocyte quantity. The problem of low yield remains a major hurdle in the field. A large number of myocytes is needed for cardiac repair as almost one billion cells is lost within an averagesized human infarct [44].

c) Genetic selection of cardiomyocytes was first introduced by Field's group [45]. The notion of linking cardiomyocyte gene expression to antibiotic resistance was further developed by others [46, 47] and adapted for use with human ESC [48]. Direct differentiation of mouse ESC into $>99 \%$ pure population of atrial cardiomyocytes was initially accomplished in the laboratory of J. Hescheler and B. Fleischmann [49]. Recently these cells became commercially available (http://www.axiogenesis.com). The purification of stem cell-derived cardiomyocytes that are obtained through genetic selection is significantly simplified. Furthermore, these cells are expected to be significantly less immunogenic, since the likelihood of developing a subpopulation of professional antigen presenting cells is reduced. However, genetically modified cells will still express endogenous allogeneic antigens. In addition, these cells will also express transgenes such as antibiotic resistance and/or fluorescent marker genes that can add to graft rejection.

d) Non-genetic enrichment of PSC-derived cardiomyocytes is a focus of recent study [50]. This method is based on non-genetic fluorescent labeling of subsarcomeric and intramyofibril mitochondria with tetramethylrhodamine methyl ester perchlorate (TMRM) selectively marking embryonic and neonatal rat cardiomyocytes, monkey, mouse, and human PSC-derived cardiomyocytes. The subsequent fluorescence-activated cell sorting of labeled cells resulted in highly enriched (99\%) of PSC-derived cardiomyocytes. The advantage of this approach is twofold. It offers relatively easy purification and expansion of PSC-derived cardiomyocytes without the introduction of additional transgenes and the associated risks and limitations for clinical use [50]. However, from an immunological point of view, these cells are expected to be as immunogenic as other PSCderived cardiomyocytes. Therefore similar concerns will need to be addressed prior to transplantation of those cells into a recipient.

\section{Antigens Classes for Stem Cell-Derived Cardiomyocytes}

Immunological barriers, including both innate and adaptive immunity, prevent integration of transplanted allogeneic PSC-derived cardiomyocytes unless the host immune system is suppressed [51, 52]. Transplant rejection occurs due to allelic differences in the surface antigens expressed by donor and recipient. There are three distinct types of transplantation antigens: $\mathrm{ABO}$ blood group antigens, minor histocompatibility antigens and major histocompatibility complex molecules. All three are expected to be found in cell populations used to create cardiac grafts.

a) A or B blood group antigens are expressed on ESC and ESC-derived cells [53]. These antigens are critical for safe blood transfusions. They have also been shown to induce rejection of solid organ transplants [54]. This immunological barrier can be avoided by isolating the starting material (i.e., embryonic stem cells) from blastocysts of an O-type blood group donor.

b) Minor histocompatibility $(\mathrm{mH})$ antigens are polymorphic peptides that are presented on the cell surface by major histocompatibility complex class I or II molecules [55]. These peptides are derived from cellular proteins encoded by different alleles of autosomal genes or by genes of the $\mathrm{Y}$ chromosome [56]. The minor histocompatibility antigen of the $\mathrm{Y}$ chromosome is associated with acute rejection, and male grafts transplanted to female recipients have reduced graft survival [57]. $\mathrm{mH}$ antigens affect the processes of transplant rejection, graft-versus-host disease and the curative graft-versus-tumor effect of stem cell transplantation [58]. Although $\mathrm{mH}$ antigens are less immunogenic than major histocompatibility complex (MHC) molecules discussed below, they are still capable of initiating allograft rejection. In fact, $\mathrm{mH}$ antigens have been shown to cause graft rejection, despite MHC antigen matching between host and donor [59]. Fully addressing $\mathrm{mH}$ mismatch between host and donor can be difficult, due to the number and the extent of $\mathrm{mH}$ antigens present throughout the genome and the Y-chromosome. The gender of PSC should at least be matched with the gender of the recipient in order to prevent minor histocompatibility antigen Y-chromosome associated rejection.

c) Major histocompatibility complex (MHC) antigens. Allelic differences in major histocompatibility complex molecules are, by far, the most significant immunological barrier in organ transplantation, including ESC-derived cell transplantations. The presence of MHC class I molecules on the surface of the cells define their susceptibility to Cytotoxic T Lymphocytes and Natural Killer cells, and thus, determine the overall survival of the 
graft. The expression levels of MHC I and MHC II antigens on the surface of ESC and PSC-derived cardiomyocytes remains an active area of research [60, 61]. During cell differentiation, the expression levels of classical MHC I and II molecules are regulated by epigenetic mechanisms [62]. The low levels of MHC class-I molecules are associated with reduced expression of the transporter associated antigen processing 1 (TAP-1) and tapasin (TPN) components. These proteins are involved in the transport and load of immunogenic peptides in both hESCs and iPS cells [62]. In addition, the expression of MHC class I molecule on the cell surface is limited by the lack of beta2-microglobulin light chain. During the differentiation process, MHC class I molecules increase as compared to undifferentiated PSCs. Similarly, the levels of beta2-microglobulin, TAP-1 and TPN increase upon differentiation [62].

While the levels of MHC-I increase upon PSC differentiation to cardiomyocytes, they are less abundant compared to other cell types. Specifically, adult cardiomyocytes from healthy hearts have been shown to have low levels of MHC I antigens, and undetectable levels of MHC II antigens and adhesion molecules, such as ICAM-1, VCAM-1 and ELAM-1 $[53,63]$. It is a population of vascular endothelial cells that serves as professional antigen presenting cells within the myocardium. As such they express high levels of MHC I, MHC II and the ICAM-1 adhesion molecule. Steinman's group also identified antigen-presenting dendritic cells in mouse aorta and cardiac valves [64]. It is possible that the rejection of PSC-derived cardiac grafts can be significantly alleviated by eliminating antigen presenting cells from these grafts. However, several studies have shown that cardiac grafts benefit from being prevascularized to ensure long-term graft survival $[11,65,66]$. The fact that endothelial cells, which are used to create these vascularized grafts [66], can act as professional antigen presenting cells should be considered more closely. One conceivable strategy would be to produce mixed grafts, in which syngeneic endothelial cells are combined with a population of allogeneic PSC-derived cardiac myocytes.

As mentioned above, the levels of MHC-I in cardiomyocytes from healthy hearts are much lower compared to other cell types. Yet, MHC I expression is greatly upregulated by inflammation. Cardiac regeneration becomes necessary after significant tissue damage. This is associated with inflammation, as shown in the cases of autoimmune myocarditis, ischemia and infarction $[67,68]$. Therefore, one should expect significant unregulation of MHC I expression on the surface of PSC-derived cardiomyocytes grafted into the site of injury.
Immunogenicity of Undifferentiated and Differentiated Stem Cells

a) Cytotoxic T lymphocyte (CTL) susceptibility. Undifferentiated stem cells express low levels of MHC I molecules and lack MHC II expression [69-71]. This is consistent with findings in both human and mouse ESC. These cells, while in an undifferentiated state, fail to stimulate an allogeneic T-lymphocyte response and may even suppress $\mathrm{T}$ cell proliferation [72-74]. Yet, infiltration of T-lymphocytes is sometimes observed at the ESC injection site [75-77]. One plausible explanation for this effect is that in vivo transplantation results in ESC death, followed by the release of ESC antigens that are then taken up by professional antigen presenting cells of the host and displayed to T-lymphocytes [75].

Undifferentiated ESC have been found to have limited susceptibility to host T-cell lysis. Therefore, injection of undifferentiated ESC was initially sought as a way to regenerate local tissue. However, it was shown that the injection of undifferentiated ESC into a specific niche does not ensure direct differentiation into a specific cell type, but instead promotes heterogeneous differentiation into multiple phenotypes [52]. Therefore, most labs pre-differentiate ESC before transplantation. The main drawback of using differentiated ESC is their increasing immunogenicity (Fig. 2). This effect is explained by higher MHC class I expression at the surface of differentiated ESC-derived cells as compared to undifferentiated cells $[60,71]$. In addition, an inflammatory environment (that can be mimicked by

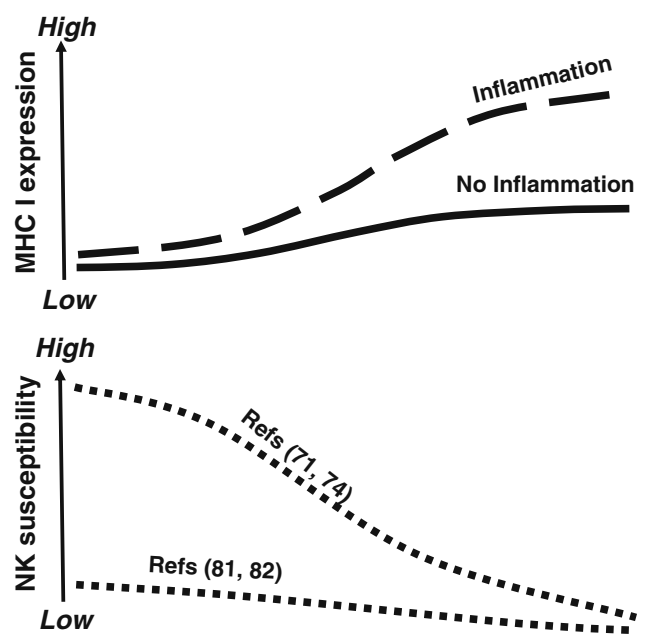

PSC Differentiation

Cardiomyocytes

Fig. 2 Cartoon illustrating trends of MHC-I expression and NK susceptibility observed upon differentiation of pluripotent stem cells 
IFN-gamma treatment) results in a more robust and fast upregulation in MHC I expression in differentiated ESC progeny as compared to undifferentiated ESC [60]. Even so, the expression level of MHC I in ESCderived cells is still less than that of adult somatic cells, consistent with their decreased susceptibility to cytotoxic T lymphocyte lysis [69, 71].

b) Natural Killer Cell susceptibility. According to the "missing-self" theory [78], Natural Killer (NK) cells destroy cells that lack self-MHC class I molecules. However, even when MHC class I levels are not diminished, alterations in the expression of ligands for stimulatory receptors are sufficient to render a target cell sensitive to NK cell mediated lysis [79]. The current paradigm is that $\mathrm{NK}$ cells express several families of inhibitory receptors, most of which are specific for MHC class I molecules, and various stimulatory receptors with diverse specificities. The balance of activating and inhibitory signals resulting from interactions with a given target cell determines whether the NK cell becomes activated to produce inflammatory cytokines, including interferon-gamma and tumor necrosis factor-alpha, and/or kill the target cells [80].

Since undifferentiated ESC express low levels of MHC-I, they should be easy targets for NK mediated lysis. However, the lack of MHC-I molecules is an essential but not sufficient factor to activate NK cells, since expression of specific proteins can modulate NK cell recognition. Today, there is controversy regarding the susceptibility of undifferentiated ESC to NK cell lysis (Fig. 2). First, it has been shown that undifferentiated human ESC are not susceptible to NK-mediated cell lysis due to low expression of ligands required for activation of NK cells [71]. This was supported by similar data in undifferentiated mouse ESC [74]. Yet, Frenzel et al. demonstrated that undifferentiated mouse ESC are readily targeted by NK cells, presumably due to the high expression level of intercellular adhesion molecule 1 (ICAM1) and moderate expression of retinoic acid early inducible-1 (RAE-1) [81]. These two molecules interact with the potent NK stimulatory receptor, NK group 2 member D (NKG2D), which may account for this recognition. The same research group has shown that in the course of differentiation, ESCderived cardiomyocytes become less susceptible to NK cell-mediated lysis by downregulating the expression of ligands required for activation of NK cells [81]. Similar results were obtained in the recent study by Dressel et al. demonstrating that not only undifferentiated ESC but also iPS cells are highly susceptible to interleukin-2 activated NK cells [82]. The NK susceptibility was again shown to be determined by NKG2D expression, and could be inhibited by the soluble form of the same molecule. Once more, the differentiation of ESC-derived and iPS cells resulted in downregulation of NKG2D expression and, consequently, diminished NK susceptibility [83]. Additional studies are needed to conclusively confirm an emerging consensus that while undifferentiated PSC cells seem to be highly susceptible to NK lysis, their derivatives, specifically differentiated cardiomyocytes, lose NK susceptibility. Whether this is true or not, potential NK susceptibility presents an obvious obstacle for the development of PSC derivatives for therapeutic purposes. Yet, the studies cited above indicate the specific molecule (i.e., NKG2D) that can be modified to avoid NK-induced rejection of PSC-derived cardiomyocytes.

c) The possible role of FAS ligand. The hallmark of immune-privileged sites is the expression of FasL molecule (CD95L). It delivers an apoptotic signal to cells expressing the Fas receptor, i.e. CD95 [84, 85]. As with NK susceptibility, it is unclear whether or not FasL expression protects PSC and their derivatives from rejection. Specifically, the expression of FasL on ESC and ESC-derived cells has been indicated as the reason for the resistance of these cells to alloreactive $\mathrm{T}$ cell- and NK cell-mediated killing in several species $[74,86]$. Another recent study has shown that bone marrow stem cells mediate immunosuppressive activity by FasL-induced killing of activated lymphocytes [87]. However, neither FasL mRNA nor FasL protein were detected in human ESC or human EB-derived cells, despite the fact that these cells were less susceptible to immune rejection than adult cells [61]. Similar results were obtained by Grinnemo et al. where FasL was not detected in a number of different human ESC lines [70]. The discrepancies between the above cited studies $[61,70,74,86,87]$ in the detection/expression of FasL on the surface of PSC and their derivatives can be explained by many factors. These include diverse types of tested PSC, a variety of methods of cell differentiation, and the stage of differentiated cells. The role of FasL expression should be better defined to learn how to manipulate the immunogenicity of PSC derived cells prior their therapeutical use.

d) Role of co-stimulatory molecules. The initiation of a comprehensive immune response is not possible without the contribution of co-stimulatory molecules. This, so-called, signal two (in contrast to signal one, which is an engagement of $\mathrm{T}$ cell receptors with the MHC-peptide complex) is antigen nonspecific and is provided by the interaction between co-stimulatory molecules expressed on the membrane of antigen presenting cell and the $\mathrm{T}$ cell. One of the best characterized co-stimulatory molecules expressed 
by $\mathrm{T}$ cells is CD28, which interacts with CD80 (B7-1) and CD86 (B7-2). Studies have demonstrated that the inhibition of CD28-mediated co-stimulation most efficiently induces unresponsiveness of cognate $\mathrm{T}$ lymphocytes [88, 89]. Many groups studied the expression levels of CD80 and CD86 on the surface of ESC and ESC-derived cells, but there is no consensus regarding the expression level. Specifically, these molecules have not been detected on the surface of human ESC and ESC-derived cells [61, 72], and rat embryonic stem cell-like cells [86]. However, low levels of CD86 but not CD80 were detected in mouse ESC and their embryoid bodies by Fairchild's group [90]. Furthermore, a subpopulation of CD80 positive cells comprising $33 \%$ of total cells was detected in mouse ESC cultures, while negligible staining was found for CD86, and CD28 [91]. When these ESC were differentiated into embryoid bodies for 12 days, both co-stimulatory proteins CD80, CD86 and their appropriate receptors, CD28 and CD154, were detected [91]. In addition, mouse neural stem/progenitor cells were found to express the co-stimulatory molecules CD80 and CD86. These molecules were shown to be differentially regulated by inflammatory and apoptotic stimuli [92]. Lastly, an analysis of the mRNA profile in undifferentiated HS237, HS293, HS306, HS346, HS362, HS363 and HS368 human ES lines detected CD80 expression, but not CD86 [70]. These numerous discrepancies regarding the presence of co-stimulatory molecules on the surface of ESC and ESC-derived cells can be explained by the species and cell type differences, as well as by the stage of differentiation and assay sensitivity. Additional systematic studies are needed to clarify the status of co-stimulatory molecules on the surface of ESC-derived cardiomyocytes.

e) Overall concerns. Several studies have found teratoma formation following transplantation of undifferentiated ESC and iPSC, which suggests escape of these cells from immune surveillance. However, in most studies, teratomas were formed only when a large dose of cells was injected [73, 77]. These teratomas were eventually rejected when observed for longer periods of time [77, 83, 93, 94]. The emerging consensus is that ESC and ESC-derivatives are sensed by the host immune system, despite the apparent lack, or very low level, of MHC I\&II and co-stimulatory molecules on their surface.

Due to the limited expression of immunogenic molecules on PSC-derived grafts, they may not be detected by traditional protein based assays, such as immunoblotting or flow cytometry. More sensitive techniques, such as quantitative real time PCR, provide evidence of mRNA expression of both heavy and light chain MHC I \& II in many ESC lines [95]. However, additional elements are needed for proper folding of these molecules on the cell surface [96]. Physiological assays, such as CTL assay, preferably done in an inflammatory environment, are needed to fully assert the immunogenicity of ESC derivatives. The importance of physiological assessment was emphasized by a recent study from Engel's group [95]. Even though the expression levels of MHC class I molecules were not detected by flow cytometry, the ESC and multipotent adult germ-line stem cells loaded with a specific peptide were lysed in a peptide-dependent manner by activated cytotoxic T-lymphocytes. In addition, new methods for CTL measurement have been devised, some of which include lactate dehydrogenase, caspase-3, granzyme $\mathrm{B}$, serine esterases, bromodeoxyuridine uptake and fluorescence detection, which are more sensitive to conventional ${ }^{51} \mathrm{Cr}$-release assays. Moreover, carboxyfluorescein diacetate
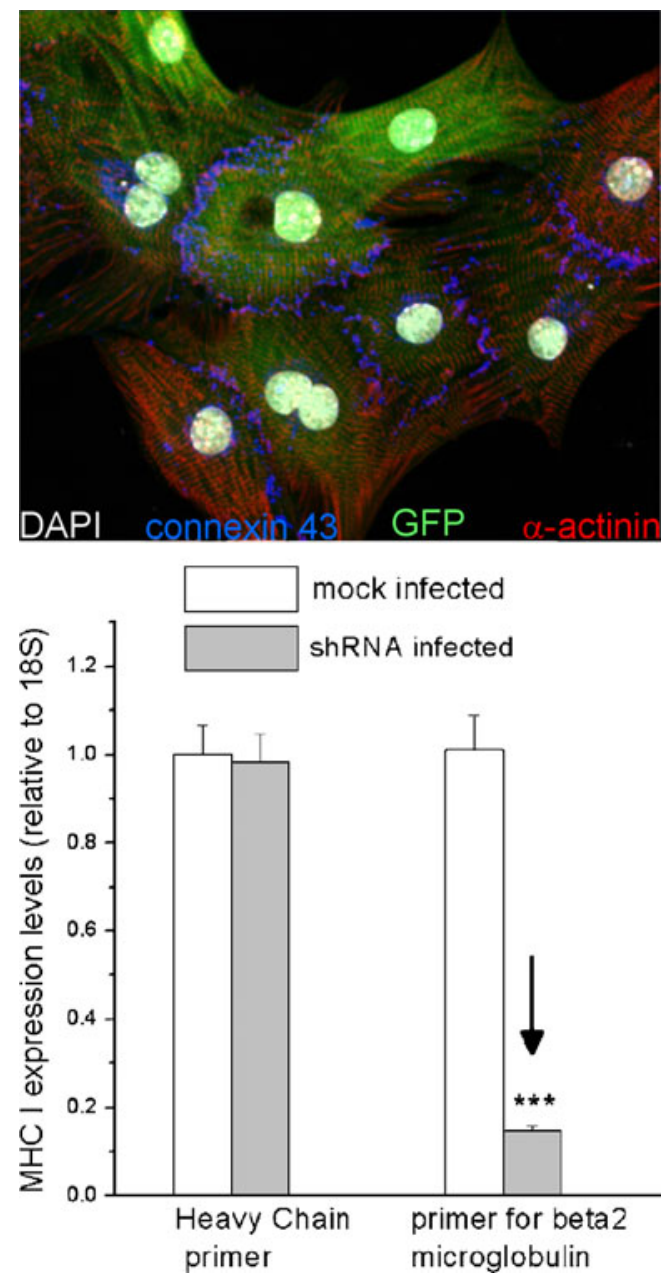

Fig. 3 Top Panel: Immunostaining of mouse ESC-derived cardiomyocytes (LONZA, catalog number XCAC-1010N). Bottom panel: ESC-derived cardiomyocytes were pretreated with interferon-gamma to elevate the baseline expression of MHC-I. RNA inhibition successfully blocked beta- 2 microglubulin expression, thus preventing formation of functional MHC-I molecules 
succinimidyl ester (CFSE) dilution has been successfully used to measure T-cell activation and division using flow cytometry both in vitro and in vivo [97]. All of these methods should be considered when assessing the immunogenicity of ESC- and iPSC-derived cardiomyocytes.

\section{Strategies to Alter the Immunogenicity of ESC and ESC-Derived Cardiomyocytes}

There are currently two lines of thought as to how immune rejection of ESC-derived grafts can be decreased. The first is tolerization of the host toward newly introduced graft antigens. This topic has been recently and extensively reviewed [51,98]. The second is to create a so-called "universal donor cell" by minimizing the immunogenic determinants of the ESC-derived graft cells. Several strategies can be used to create immunologically "invisible" ESC and their derivates.

One strategy is to overexpress FasL, a hallmark of immune-privileged sites [85]. FasL induces apoptosis of T-cells that express Fas receptor (CD95). Data suggests that the success of this strategy is organ specific. Transgenic FasL expression in pancreatic and cardiac transplants resulted in tissue damage or accelerated rejection, while FasL expression in the liver, kidney, lung, blood vessels and thyroid led to a diminished immune response [99]. Additional studies are needed to address the potential pitfalls of FasL overexpression, including the destructive effects of transplanting FasL-expressing cells into tissues that express the Fas receptor.

The second strategy is to overexpress Serpin 6 (Serine protease inhibitor 6). It is an endogenous inhibitor of the CTL-derived cytotoxic effector molecule-granzyme B. Utermöhlen's group demonstrated that ESC resist CTLmediated lysis via high-level expression of Serpin 6 [100]. Therefore, maintaining or upregulating levels of Serpin 6 in ESC-derived cardiomyocytes may diminish their susceptibility to rejection.

Finally, the amount of MHC-I molecules could be severely reduced. MHC-I negative ESC can be derived from the embryos of $\beta 2$-microglobulin knockout mice [101]. Although downregulation of MHC expression in traditional tissue and organ transplantation is not novel, rapid development of new techniques such as RNA interference provides significant improvements and the flexibility to target a specific gene. Individual heavy and light chains of MHC class I molecule were successfully manipulated in many studies [102-105]. Combining RNA inhibition technology with the derivation of differentiated platelets from progenitor cells was recently shown to be feasible in the generation of MHC I deficient cells [106]. Similarly, RNA inhibition technology could also be used to achieve this effect in already differentiated ESC-derived cardiac myocytes (Fig. 3). In the absence of $\beta 2$ microglobulin protein, functional MHC-I molecules will not be formed. This will result in low levels of MHC-I in ESC and their derivatives, including cardiomyocytes. Considering that MHC-II expression is absent from ESCderived cardiomyocytes, these cells should have minimal immunogenicity. There will still be elements, such as minor histocompatibility $(\mathrm{mH})$ antigens or residual MHC expression that can cause problems [107, 108]. In addition, low levels of MHC I expression can also activate NK mediated cell killing. However, as we discussed above, NK lysis can be addressed by overexpression of NK inhibitory receptors on the surface of PSC-derived cardiomyocytes. All-in-all, more studies are needed to address these concerns. Yet, the creation of a universal donor ESC line that can be differentiated to a desired cell type and transplanted to any individual with minimal risk of graft rejection remains an exciting therapeutic goal.

Acknowledgements Financial support by the National Institutes of Health (HL076722 to NS, F32HL087529 to ZK and Mid-Atlantic American Heart Association 0715335U to NGP) is gratefully acknowledged. We also would like to thank Axiogenesis $A G$ for supplying the mouse ESC-derived myocytes.

Conflicts of Interest The authors declare no potential conflicts of interest.

Open Access This article is distributed under the terms of the Creative Commons Attribution Noncommercial License which permits any noncommercial use, distribution, and reproduction in any medium, provided the original author(s) and source are credited.

\section{References}

1. Zak, R. (1974). Development and proliferative capacity of cardiac muscle cells. Circulation Research, 35(suppl II), 17-26.

2. Bergmann, O., Bhardwaj, R. D., Bernard, S., et al. (2009). Evidence for cardiomyocyte renewal in humans. Science, 324, 98-102.

3. Pasumarthi, K. B., \& Field, L. J. (2002). Cardiomyocyte cell cycle regulation. Circulation Research, 90, 1044-1054.

4. Hodgson, D. M., Behfar, A., Zingman, L. V., et al. (2004). Stable benefit of embryonic stem cell therapy in myocardial infarction. American Journal of Physiology. Heart and Circulatory Physiology, 287, H471-H479.

5. Caspi, O., Huber, I., Kehat, I., et al. (2007). Transplantation of human embryonic stem cell-derived cardiomyocytes improves myocardial performance in infarcted rat hearts. Journal of the American College of Cardiology, 50, 1884-1893.

6. Tomescot, A., Leschik, J., Bellamy, V., et al. (2007). Differentiation in vivo of cardiac committed human embryonic stem cells in postmyocardial infarcted rats. Stem Cells, $25,2200-2205$.

7. Singla, D. K., Hacker, T. A., Ma, L., et al. (2006). Transplantation of embryonic stem cells into the infarcted mouse heart: formation of multiple cell types. Journal of Molecular and Cellular Cardiology, 40, 195-200. 
8. Blank, A. C., van Veen, T. A., Jonsson, M. K., et al. (2009). Rewiring the heart: stem cell therapy to restore normal cardiac excitability and conduction. Current Stem Cell Research \& Therapy, 4, 23-33.

9. Rosen, M. R., Brink, P. R., Cohen, I. S., \& Robinson, R. B. (2004). Genes, stem cells and biological pacemakers. Cardiovascular Research, 64, 12-23.

10. Laflamme, M. A., Zbinden, S., Epstein, S. E., \& Murry, C. E. (2007). Cell-based therapy for myocardial ischemia and infarction: pathophysiological mechanisms. Annual Review of Pathology, 2, 307-339.

11. Stevens, K. R., Kreutziger, K. L., Dupras, S. K., et al. (2009). Physiological function and transplantation of scaffold-free and vascularized human cardiac muscle tissue. Proceedings of the National Academy of Sciences of the United States of America, 106, 16568-16573.

12. Hosoda, T., D’Amario, D., Cabral-Da-Silva, M. C., et al. (2009). Clonality of mouse and human cardiomyogenesis in vivo. Proceedings of the National Academy of Sciences of the United States of America, 106, 17169-17174.

13. Hwang, W. S., Ryu, Y. J., Park, J. H., et al. (2004). Evidence of a pluripotent human embryonic stem cell line derived from a cloned blastocyst. Science, 303, 1669-1674.

14. Davis, D. R., Ruckdeschel Smith, R., \& Marban, E. (2010). Human cardiospheres are a source of stem cells with cardiomyogenic potential. Stem Cells, 28, 903-904.

15. Nishiyama, N., Miyoshi, S., Hida, N., et al. (2007). The significant cardiomyogenic potential of human umbilical cord blood-derived mesenchymal stem cells in vitro. Stem Cells, 25 , 2017-2024.

16. Yu, J., Vodyanik, M. A., Smuga-Otto, K., et al. (2007). Induced pluripotent stem cell lines derived from human somatic cells. Science, 318, 1917-1920.

17. Takahashi, K., Tanabe, K., Ohnuki, M., et al. (2007). Induction of pluripotent stem cells from adult human fibroblasts by defined factors. Cell, 131, 861-872.

18. Takahashi, K., \& Yamanaka, S. (2006). Induction of pluripotent stem cells from mouse embryonic and adult fibroblast cultures by defined factors. Cell, 126, 663-676.

19. Barile, L., Messina, E., Giacomello, A., \& Marban, E. (2007). Endogenous cardiac stem cells. Progress in Cardiovascular Diseases, 50, 31-48.

20. Bearzi, C., Rota, M., Hosoda, T., et al. (2007). Human cardiac stem cells. Proceedings of the National Academy of Sciences of the United States of America, 104, 14068-14073.

21. Linke, A., Muller, P., Nurzynska, D., et al. (2005). Stem cells in the dog heart are self-renewing, clonogenic, and multipotent and regenerate infarcted myocardium, improving cardiac function. Proceedings of the National Academy of Sciences of the United States of America, 102, 8966-8971.

22. Martin, G. R. (1981). Isolation of a pluripotent cell line from early mouse embryos cultured in medium conditioned by teratocarcinoma stem cells. Proceedings of the National Academy of Sciences of the United States of America, 78, 7634-7638.

23. Evans, M. J., \& Kaufman, M. H. (1981). Establishment in culture of pluripotential cells from mouse embryos. Nature, 292, 154-156.

24. Thomson, J. A., Itskovitz-Eldor, J., Shapiro, S. S., et al. (1998) Embryonic stem cell lines derived from human blastocysts. Science, $282,1145-1147$

25. Reubinoff, B. E., Pera, M. F., Fong, C. Y., Trounson, A., \& Bongso, A. (2000). Embryonic stem cell lines from human blastocysts: somatic differentiation in vitro. Nature Biotechnology, $18,399-404$.

26. Winkel, G. K., \& Pedersen, R. A. (1988). Fate of the inner cell mass in mouse embryos as studied by microinjection of lineage tracers. Developmental Biology, 127, 143-156.
27. Odorico, J. S., Kaufman, D. S., \& Thomson, J. A. (2001). Multilineage differentiation from human embryonic stem cell lines. Stem Cells, 19, 193-204.

28. Zhang, J., Wilson, G. F., Soerens, A. G., et al. (2009). Functional cardiomyocytes derived from human induced pluripotent stem cells. Circulation Research, 104, e30-e41.

29. Woltjen, K., Michael, I. P., Mohseni, P., et al. (2009). piggyBac transposition reprograms fibroblasts to induced pluripotent stem cells. Nature, 458, 766-770.

30. Kaji, K., Norrby, K., Paca, A., Mileikovsky, M., Mohseni, P., \& Woltjen, K. (2009). Virus-free induction of pluripotency and subsequent excision of reprogramming factors. Nature, 458, 771-775.

31. Warren, L., Manos, P. D., Ahfeldt, T., et al. (2010). Highly Efficient Reprogramming to pluripotency and directed differentiation of human cells with synthetic modified mRNA. Cell Stem Cell, 7, 1-13.

32. Wobus, A. M., Wallukat, G., \& Hescheler, J. (1991). Pluripotent mouse embryonic stem cells are able to differentiate into cardiomyocytes expressing chronotropic responses to adrenergic and cholinergic agents and $\mathrm{Ca} 2+$ channel blockers. Differentiation, 48, 173-182.

33. Gepstein, L. (2002). Derivation and potential applications of human embryonic stem cells. Circulation Research, 91, 866-876.

34. Kehat, I., Kenyagin-Karsenti, D., Snir, M., et al. (2001). Human embryonic stem cells can differentiate into myocytes with structural and functional properties of cardiomyocytes. Journal of Clinical Investigation, 108, 407-414.

35. Doetschman, T. C., Eistetter, H., Katz, M., Schmidt, W., \& Kemler, R. (1985). The in vitro development of blastocystderived embryonic stem cell lines: formation of visceral yolk sac, blood islands and myocardium. Journal of Embryology and Experimental Morphology, 87, 27-45.

36. Boheler, K. R., Czyz, J., Tweedie, D., Yang, H. T., Anisimov, S. V., \& Wobus, A. M. (2002). Differentiation of pluripotent embryonic stem cells into cardiomyocytes. Circulation Research, 91, 189-201.

37. Wobus, A. M., \& Guan, K. (1998). Embryonic stem cell-derived cardiac differentiation: modulation of differentiation and "lossof-function" analysis in vitro. Trends in Cardiovascular Medicine, 8, 64-74.

38. Karabekian, Z., Gillum, N. D., Wong, E. W., \& Sarvazyan, N. (2009). Effects of N-cadherin overexpression on the adhesion properties of embryonic stem cells. Cell Adhesion \& Migration, 3, 305-310.

39. Laflamme, M. A., Chen, K. Y., Naumova, A. V., et al. (2007). Cardiomyocytes derived from human embryonic stem cells in pro-survival factors enhance function of infarcted rat hearts. Nature Biotechnology, 25, 1015-1024.

40. Takahashi, T., Lord, B., Schulze, P. C., et al. (2003). Ascorbic acid enhances differentiation of embryonic stem cells into cardiac myocytes. Circulation, 107, 1912-1916.

41. Wobus, A. M., Kaomei, G., Shan, J., et al. (1997). Retinoic acid accelerates embryonic stem cell-derived cardiac differentiation and enhances development of ventricular cardiomyocytes. Journal of Molecular and Cellular Cardiology, 29, 1525-1539.

42. Behfar, A., Perez-Terzic, C., Faustino, R. S., et al. (2007). Cardiopoietic programming of embryonic stem cells for tumorfree heart repair. The Journal of Experimental Medicine, 204, 405-420.

43. Cerdan, C., Hong, S. H., \& Bhatia, M. (2007). Formation and hematopoietic differentiation of human embryoid bodies by suspension and hanging drop cultures. Current Protocols in Stem Cell Biology Chapter 1:Unit 1D.2.

44. Murry, C. E., Reinecke, H., \& Pabon, L. M. (2006). Regeneration gaps: observations on stem cells and cardiac repair. Journal of the American College of Cardiology, 47, 1777-1785. 
45. Klug, M. G., Soonpaa, M. H., Koh, G. Y., \& Field, L. J. (1996). Genetically selected cardiomyocytes from differentiating embronic stem cells form stable intracardiac grafts. Journal of Clinical Investigation, 98, 216-224.

46. Zandstra, P. W., Bauwens, C., Yin, T., et al. (2003). Scalable production of embryonic stem cell-derived cardiomyocytes. Tissue Engineering, 9, 767-778.

47. Kita-Matsuo, H., Barcova, M., Prigozhina, N., et al. (2009). Lentiviral vectors and protocols for creation of stable hESC lines for fluorescent tracking and drug resistance selection of cardiomyocytes. PLoS ONE, 4, e5046.

48. Anderson, D., Self, T., Mellor, I. R., Goh, G., Hill, S. J., \& Denning, C. (2007). Transgenic enrichment of cardiomyocytes from human embryonic stem cells. Molecular Therapy, 15, 2027-2036.

49. Kolossov, E., Bostani, T., Roell, W., et al. (2006). Engraftment of engineered ES cell-derived cardiomyocytes but not BM cells restores contractile function to the infarcted myocardium. The Journal of Experimental Medicine, 203, 2315-2327.

50. Hattori, F., Chen, H., Yamashita, H., et al. (2010). Nongenetic method for purifying stem cell-derived cardiomyocytes. Nature Methods, 7, 61-66.

51. Lui, K. O., Waldmann, H., \& Fairchild, P. J. (2009). Embryonic stem cells: overcoming the immunological barriers to cell replacement therapy. Current Stem Cell Research \& Therapy, $4,70-80$.

52. He, Q., Trindade, P. T., Stumm, M., et al. (2009). Fate of undifferentiated mouse embryonic stem cells within the rat heart: role of myocardial infarction and immune suppression. Journal of Cellular and Molecular Medicine, 13, 188-201.

53. Molne, J., Bjorquist, P., Andersson, K., et al. (2008). Blood group $\mathrm{ABO}$ antigen expression in human embryonic stem cells and in differentiated hepatocyte- and cardiomyocyte-like cells. Transplantation, 86, 1407-1413.

54. Chan, J. H., Dua, H. S., Powell-Richards, A., Jones, D. R., \& Harris, I. M. (2001). Effect of ABO blood group mismatching on corneal epithelial cells: an in vitro study. The British Journal of Ophthalmology, 85, 1104-1109.

55. Simpson, E., Roopenian, D., \& Goulmy, E. (1998). Much ado about minor histocompatibility antigens. Immunology Today, 19, $108-112$.

56. Spierings, E., \& Goulmy, E. (2005). Expanding the immunotherapeutic potential of minor histocompatibility antigens. Journal of Clinical Investigation, 115, 3397-3400.

57. Dierselhuis, M., \& Goulmy, E. (2009). The relevance of minor histocompatibility antigens in solid organ transplantation. Current Opinion in Organ Transplantation, 14, 419-425.

58. Spierings, E., Hendriks, M., Absi, L., et al. (2007). Phenotype frequencies of autosomal minor histocompatibility antigens display significant differences among populations. PLoS Genetics, 3 , e103.

59. Fairchild, P. J., Robertson, N. J., Minger, S. L., \& Waldmann, H. (2007). Embryonic stem cells: protecting pluripotency from alloreactivity. Current Opinion in Immunology, 19, 596-602.

60. Boyd, A. S., \& Wood, K. J. (2009). Variation in MHC expression between undifferentiated mouse ES cells and ES cell-derived insulin-producing cell clusters. Transplantation, 87, 1300-1304.

61. Drukker, M., Katchman, H., Katz, G., et al. (2006). Human embryonic stem cells and their differentiated derivatives are less susceptible to immune rejection than adult cells. Stem Cells, 24, 221-229.

62. Suarez-Alvarez, B., Rodriguez, R. M., Calvanese, V., et al. (2010). Epigenetic mechanisms regulate MHC and antigen processing molecules in human embryonic and induced pluripotent stem cells. PLoS ONE, 5, e10192.
63. Ardehali, A., Laks, H., Drinkwater, D. C., Jr, et al. (1993). Expression of major histocompatibility antigens and vascular adhesion molecules on human cardiac allografts preserved in University of Wisconsin solution. Journal of Heart and Lung Transplantation, 12:1044,51;1051-1052.

64. Choi, J. H., Do, Y., Cheong, C., et al. (2009). Identification of antigen-presenting dendritic cells in mouse aorta and cardiac valves. The Journal of Experimental Medicine, 206, 497-505.

65. Zimmermann, W. H., Melnychenko, I., Wasmeier, G., et al. (2006). Engineered heart tissue grafts improve systolic and diastolic function in infarcted rat hearts. Natural Medicines, 12, $452-458$.

66. Maidhof, R., Marsano, A., Lee, E. J., \& Vunjak-Novakovic, G. (2010). Perfusion seeding of channeled elastomeric scaffolds with myocytes and endothelial cells for cardiac tissue engineering. Biotechnology Progress, 26, 565-572.

67. Yoshida, T., Hanawa, H., Toba, K., et al. (2005). Expression of immunological molecules by cardiomyocytes and inflammatory and interstitial cells in rat autoimmune myocarditis. Cardiovascular Research, 68, 278-288.

68. Frantz, S., Bauersachs, J., \& Ertl, G. (2009). Post-infarct remodelling: contribution of wound healing and inflammation. Cardiovascular Research, 81, 474-481.

69. Ladhoff, J., Bader, M., Brosel, S., et al. (2009). Low immunogenicity of endothelial derivatives from rat embryonic stem celllike cells. Cell Research, 19, 507-518.

70. Grinnemo, K. H., Kumagai-Braesch, M., Mansson-Broberg, A., et al. (2006). Human embryonic stem cells are immunogenic in allogeneic and xenogeneic settings. Reproductive Biomedicine Online, 13, 712-724.

71. Drukker, M., Katz, G., Urbach, A., et al. (2002). Characterization of the expression of MHC proteins in human embryonic stem cells. Proceedings of the National Academy of Sciences of the United States of America, 99, 9864-9869.

72. Li, L., Baroja, M. L., Majumdar, A., et al. (2004). Human embryonic stem cells possess immune-privileged properties. Stem Cells, 22, 448-456.

73. Koch, C. A., Geraldes, P., \& Platt, J. L. (2008). Immunosuppression by embryonic stem cells. Stem Cells, 26, 89-98.

74. Bonde, S., \& Zavazava, N. (2006). Immunogenicity and engraftment of mouse embryonic stem cells in allogeneic recipients. Stem Cells, 24, 2192-2201.

75. Swijnenburg, R. J., Schrepfer, S., Cao, F., et al. (2008). In vivo imaging of embryonic stem cells reveals patterns of survival and immune rejection following transplantation. Stem Cells and Development, 17, 1023-1029.

76. Kofidis, T., deBruin, J. L., Tanaka, M., et al. (2005). They are not stealthy in the heart: embryonic stem cells trigger cell infiltration, humoral and T-lymphocyte-based host immune response. European Journal of Cardiothoracic Surgery, 28, 461-466.

77. Nussbaum, J., Minami, E., Laflamme, M. A., et al. (2007). Transplantation of undifferentiated murine embryonic stem cells in the heart: teratoma formation and immune response. The FASEB Journal, 21, 1345-1357.

78. Raulet, D. H. (2006). Missing self recognition and self tolerance of natural killer (NK) cells. Seminars in Immunology, 18, 145-150.

79. Manilay, J. O., \& Sykes, M. (1998). Natural killer cells and their role in graft rejection. Current Opinion in Immunology, 10, 532538.

80. Joncker, N. T., \& Raulet, D. H. (2008). Regulation of NK cell responsiveness to achieve self-tolerance and maximal responses to diseased target cells. Immunological Reviews, 224, 85-97.

81. Frenzel, L. P., Abdullah, Z., Kriegeskorte, A. K., et al. (2009). Role of natural-killer group 2 member D ligands and intercellular adhesion molecule 1 in natural killer cell-mediated lysis of 
murine embryonic stem cells and embryonic stem cell-derived cardiomyocytes. Stem Cells, 27, 307-316.

82. Dressel, R., Nolte, J., Elsner, L., et al. (2010). Pluripotent stem cells are highly susceptible targets for syngeneic, allogeneic, and xenogeneic natural killer cells. The FASEB Journal, 24, 2164-2177.

83. Dressel, R., Schindehütte, J., Kuhlmann, T., et al. (2008). The tumorigenicity of mouse embryonic stem cells and in vitro differentiated neuronal cells is controlled by the recipients' immune response. PLOS ONE, 3, e2622.

84. Steller, H. (1995). Mechanisms and genes of cellular suicide. Science, 267, 1445-1449.

85. Griffith, T. S., Brunner, T., Fletcher, S. M., Green, D. R., \& Ferguson, T. A. (1995). Fas ligand-induced apoptosis as a mechanism of immune privilege. Science, 270, 1189-1192.

86. Fandrich, F., Lin, X., Chai, G. X., et al. (2002). Preimplantation-stage stem cells induce long-term allogeneic graft acceptance without supplementary host conditioning. Natural Medicines, 8, 171-178.

87. Mazar, J., Thomas, M., Bezrukov, L., et al. (2009). Cytotoxicity mediated by the Fas ligand (FasL)-activated apoptotic pathway in stem cells. The Journal of Biological Chemistry, 284, 22022-22028.

88. Bachmann, M. F., Speiser, D. E., Mak, T. W., \& Ohashi, P. S. (1999). Absence of co-stimulation and not the intensity of TCR signaling is critical for the induction of $\mathrm{T}$ cell unresponsiveness in vivo. European Journal of Immunology, 29, 2156-2166.

89. Boggs, J. M., Chang, N. H., Goundalkar, A., \& Hashim, G. A. (1992). Stimulation or tolerization of an anti-myelin basic protein $\mathrm{T}$ lymphocyte line with membrane fragments from antigen presenting cells. Cellular Immunology, 143, 23-40.

90. Robertson, N. J., Brook, F. A., Gardner, R. L., Cobbold, S. P., Waldmann, H., \& Fairchild, P. J. (2007). Embryonic stem cell-derived tissues are immunogenic but their inherent immune privilege promotes the induction of tolerance. Proceedings of the National Academy of Sciences of the United States of America, 104, 20920 20925.

91. Ling, V., Munroe, R. C., Murphy, E. A., \& Gray, G. S. (1998). Embryonic stem cells and embryoid bodies express lymphocyte costimulatory molecules. Experimental Cell Research, 241, 55-65.

92. Imitola, J., Comabella, M., Chandraker, A. K., et al. (2004). Neural stem/progenitor cells express costimulatory molecules that are differentially regulated by inflammatory and apoptotic stimuli. The American Journal of Pathology, 164, 1615-1625.

93. Gutierrez-Aranda, I., Ramos-Mejia, V., Bueno, C., et al. (2010). Human induced pluripotent stem cells develop teratoma more efficiently and faster than human embryonic stem cells regardless the site of injection. Stem Cells, 28, 1568-1570.

94. Swijnenburg, R. J., Tanaka, M., Vogel, H., et al. (2005). Embryonic stem cell immunogenicity increases upon differentiation after transplantation into ischemic myocardium. Circulation, 112, I166-I172.
95. Dressel, R., Guan, K., Nolte, J., et al. (2009). Multipotent adult germ-line stem cells, like other pluripotent stem cells, can be killed by cytotoxic $\mathrm{T}$ lymphocytes despite low expression of major histocompatibility complex class I molecules. Biology Direct, 4, 31.

96. Bikoff, E. K., Jaffe, L., Ribaudo, R. K., Otten, G. R., Germain, R. N., \& Robertson, E. J. (1991). MHC class I surface expression in embryo-derived cell lines inducible with peptide or interferon. Nature, 354, 235-238.

97. Plebanski, M., Katsara, M., Sheng, K. C., Xiang, S. D., \& Apostolopoulos, V. (2010). Methods to measure T-cell responses. Expert Reviews of Vaccines, 9, 595-600.

98. Grinnemo, K. H., Sylven, C., Hovatta, O., Dellgren, G., \& Corbascio, M. (2008). Immunogenicity of human embryonic stem cells. Cell and Tissue Research, 331, 67-78.

99. Green, D. R., \& Ferguson, T. A. (2001). The role of Fas ligand in immune privilege. Nature Reviews. Molecular Cell Biology, 2, 917-924.

100. Abdullah, Z., Saric, T., Kashkar, H., et al. (2007). Serpin-6 expression protects embryonic stem cells from lysis by antigenspecific CTL. Journal of Immunology, 178, 3390-3399.

101. Zijlstra, M., Bix, M., Simister, N. E., Loring, J. M., Raulet, D. H., \& Jaenisch, R. (1990). Beta 2-microglobulin deficient mice lack CD4-8+ cytolytic T cells. Nature, 344, 742-746.

102. Haga, K., Lemp, N. A., Logg, C. R., et al. (2006). Permanent, lowered HLA class I expression using lentivirus vectors with shRNA constructs: averting cytotoxicity by alloreactive $\mathrm{T}$ lymphocytes. Transplantation Proceedings, 38, 3184-3188.

103. Figueiredo, C., Horn, P. A., Blasczyk, R., \& Seltsam, A. (2007). Regulating MHC expression for cellular therapeutics. Transfusion, 47, 18-27.

104. Figueiredo, C., Seltsam, A., \& Blasczyk, R. (2006). Class-, gene-, and group-specific HLA silencing by lentiviral shRNA delivery. Journal of Molecular Medicine, 84, 425-437.

105. Horn, P. A., Figueiredo, C., \& Kiem, H. P. (2007). Gene therapy in the transplantation of allogeneic organs and stem cells. Current Gene Therapy, 7, 458-468.

106. Figueiredo, C., Goudeva, L., Horn, P. A., Eiz-Vesper, B., Blasczyk, R., \& Seltsam, A. (2010). Generation of HLA-deficient platelets from hematopoietic progenitor cells. Transfusion, 50, 1690-1701.

107. Grusby, M. J., Auchincloss, H., Jr., Lee, R., et al. (1993). Mice lacking major histocompatibility complex class I and class II molecules. Proceedings of the National Academy of Sciences of the United States of America, 90, 3913-3917.

108. Dierich, A., Chan, S. H., Benoist, C., \& Mathis, D. (1993). Graft rejection by $\mathrm{T}$ cells not restricted by conventional major histocompatibility complex molecules. European Journal of Immunology, 23, 2725-2728. 